\title{
Predictors of Paediatric Influenza-like-Illness Severity During the COVID-19 Pandemic
}

\author{
George Porter ${ }^{1}$ and Stephen Owens ${ }^{2}$ \\ ${ }^{1}$ Newcastle University Faculty of Medical Sciences \\ ${ }^{2}$ Great North Children's Hospital
}

October 16, 2020

Predictors of Paediatric Influenza-like-Illness Severity During the COVID-19 Pandemic

*George James Porter ${ }^{1}$ and Stephen Owens ${ }^{2,3}$

1. Faculty of Medical Sciences, Newcastle University, Framlington Place, Newcastle Upon Tyne, United Kingdom.

2. Department of Paediatric Immunology and Infectious Diseases, Great North Children's Hospital, Newcastle upon Tyne Hospital NHS Foundation Trust, Newcastle upon Tyne, United Kingdom.

3. Population Health Sciences Institute, Newcastle University, Framlington Place, Newcastle Upon Tyne, United Kingdom.

*Indicates the corresponding author, correspondence to: g.porter2@newcastle.ac.uk

Word Count: 807

Respiratory viral illness is a common cause of paediatric admission in the UK. It is estimated that In an average winter in the UK, $2.4 \%$ of children under five will have a respiratory illness attributable to Influenza A alone.(1) Despite the importance of viral respiratory infection, the predictive value of symptoms, co-morbidities and viral isolation are debated.(2) For example, in an American study of 241 children presenting to hospital with influenza-like-illness (ILI) the risk of severe complications was only increased by neurological/neuromuscular disease.(3) Meanwhile, a similar British study analysing 265 paediatric H1N1positive ILI patients each with 2 virus-negative ILI controls found that chronic lung disease, cerebral palsy, chest retractions, tachycardia, dehydration and oxygen requirement predicted adverse outcome across the two groups.(4)

The ongoing COVID-19 pandemic has shown that surges of acute respiratory infections must be planned for by the health service. Therefore, clearer definition of the early indicators of high-risk patients could be of considerable benefit regarding resource allocation and risk stratification. Equally, few studies have taken into account demographic data (including age and ethnicity), co-morbidities and symptoms and signs at presentation simultaneously.(5) We therefore conducted a retrospective study to help identify the predictors of severity of ILI in the North East of England during the height of the coronavirus pandemic.

We defined ILI as the presence of fever [?] $37.8^{\circ} \mathrm{C}$ and one other upper respiratory symptom. A cohort of children hospitalised with ILI in Newcastle-Upon-Tyne hospitals between 01/03/2020 and 05/05/2020 was identified from trust records. Information on symptoms at presentation, past medical history, demographics and outcomes was extracted. Disease severity was approximated by admission length, oxygen usage and intensive care unit (ITU) admission. Regression modelling identified variables which impacted these outcomes. 


\begin{tabular}{lllll}
\hline Variable & Odds Ratio & Standard Error & P Value & $95 \%$ Confidence interval \\
\hline ITU Admission & & & & \\
Coryza & 0.3292 & 0.1852 & 0.048 & $0.109-0.992$ \\
Difficulty Breathing & 6.1567 & 4.3645 & 0.010 & $1.534-24.70$ \\
Seizures & 10.335 & 8.2837 & 0.004 & $2.148-49.72$ \\
Cardiac Co-Morbidity & 6.3733 & 4.1067 & 0.004 & $1.803-22.53$ \\
Oxygen Usage & & & & \\
Difficulty Breathing & 15.029 & 8.0148 & $<0.001$ & $5.284-42.74$ \\
Cardiac co-morbidity & 14.838 & 17.760 & 0.024 & $1.421-155.0$ \\
Genetic co-morbidity & 14.742 & 18.048 & 0.028 & $1.338-162.4$ \\
Male Gender & 3.215 & 1.7317 & 0.030 & $1.119-9.240$ \\
Admission Length (days) & Coefficient & & & \\
Lethargy & -5.436 & 2.504 & 0.032 & $-10.39--0.476$ \\
Coryza & -4.788 & 2.375 & 0.046 & $-9.492--0.084$ \\
Sore throat & -11.60 & 4.036 & 0.005 & $-19.59--3.068$ \\
Cardiac Co-Morbidity & 11.06 & 3.460 & 0.002 & $4.207-17.91$ \\
Oncology/Haematology Co-Morbidity & 14.56 & 3.274 & $>0.001$ & $8.081-21.05$ \\
\hline
\end{tabular}

123 patients were included and their median age was 3.5 years. Difficulty breathing, seizures before or during admission and congenital cardiac defect significantly $(p<0.05)$ increased the likelihood of ITU admission. The likelihood of oxygen requirement was significantly increased by difficulty breathing on presentation, cardiac defect, genetic co-morbidity and male gender. Admission length was significantly increased by cardiac defect and Oncological/Haematological co-morbidity. Table 1 shows the regression models.

Table :Table showing the regression model summaries illustrating the clinical parameters which affected the three outcome variables in our sample.

Eighty-seven patients were tested for respiratory viruses, $33 \%$ of which had at least 1 isolated. Rhinovirus was the most commonly detected virus at $14.94 \%$ followed by Adenovirus at $12.64 \%$. On univariate analysis, viral isolation significantly increased the likelihood of ITU admission and the length of admission. However, addition of any other variables to the univariate models resulted in virology becoming non-significant, implying much of the association was due to confounders like co-morbidity. Similarly, individual viruses including COVID-19 did not impact outcomes apart from adenovirus, which occurred in only 10 cases but was significantly associated with ITU admission.

Our study identified several predictors of morbidity in our cohort of severe paediatric ILI patients. Specifically, given that cardiac defects significantly worsened all outcome measures, efforts must be made to continue to deliver the surgical care necessary for these patients. Many surgical units suspended or postponed a high number of non-urgent operations during the pandemic. Our study implies that further research into balancing the risks and benefits of such measures in particular groups, such as children with cardiac defects, may be justified.

While univariate analysis showed that positive virology increased admission length and the likelihood of PICU admission, taking into account any other severity predictors resulted in this association becoming non-significant. This suggests that any association in our sample was heavily modified by co-morbidities. Several similar studies to ours have also concluded that viral isolation is of little value.(3)

However, our study was conducted outside peak respiratory virus season and our sample's viral positivity rate was low (33\%) compared to studies which found that viral isolation impacted disease severity.(5) It is therefore possible that virology becomes an important predictor of severity in high-prevalence environments. Regardless, virology tests are uncomfortable for patients and take up clinical and laboratory time, so research into their impact on a patient's treatment in low-prevalence environments is necessary. 
Our study is subject to some limitations. One will note the large confidence intervals around some of the ORs and coefficients in the study. All models were evaluated for collinearity, specification error and goodness-offit. None of these tests identified any problems with the model, implying that the large odds ratios were due to sample size. Therefore we have identified clinical parameters which affect viral respiratory illness outcome in our sample as well as effect direction, but we cannot precisely estimate effect size.

In conclusion, this study identified several groups of children which exhibit disproportionately severe ILI. As the social distancing measures present during the study period are lifted it is likely that there will be a rise in infective presentations. These groups should therefore be given special consideration by public health policy makers. Positive virology in general had little predictive value, therefore research into the necessity of these tests is required.

1. Fleming DM, Taylor RJ, Haguinet F, et al. Influenza-attributable burden in United Kingdom primary care. Epidemiol Infect . 2016;144(3):537-47.

2. Tregoning JS, Schwarze J. Respiratory viral infections in infants: causes, clinical symptoms, virology, and immunology. Clin Microbiol Rev . 2010;23(1):74-98.

3. Mistry RD, Fischer JB, Prasad PA, et al. Severe Complications in Influenza-like Illnesses. Pediatrics . 2014;134(3):e684-e90.

4. Dalziel SR, Thompson JMD, Macias CG, et al. Predictors of severe H1N1 infection in children presenting within Pediatric Emergency Research Networks (PERN): retrospective case-control study. BMJ . 2013;347:f4836.

5. Shi T, McLean K, Campbell H, Nair H. Aetiological role of common respiratory viruses in acute lower respiratory infections in children under five years: A systematic review and meta-analysis. $J$ Glob Health . 2015;5(1):010408. 